\title{
The association of sidewalk walkability and physical disorder with area-level race and poverty
}

\author{
Cheryl M Kelly, Mario Schootman, Elizabeth A Baker, Ellen K Barnidge, Amanda Lemes
}

J Epidemiol Community Health 2007;61:978-983. doi: 10.1136/jech.2006.054775

See end of article for authors' affiliations

Correspondence to: Cheryl M Kelly, Transtria, 3525 Watson Road, St Louis, MO 63139, USA; kellycm@gmail.com

Accepted 3 January 2007

\begin{abstract}
Introduction: There are significant differences in physical inactivity in various geographical areas and among demographic groups. Previous research suggests that walking is the most common form of physical activity; however, not all built environments support walking for recreational or transportation purposes.

Objective: The purpose of this study was to assess the extent to which area-level factors, poverty rate and racial distribution, are associated with aspects of the street-scale environment (i.e. sidewalk walkability and physical disorder) using community audits.

Methods: Street segments were randomly selected from 210 block groups. Pairs of trained auditors walked each street segment using an audit tool designed to capture aspects of the street environment. Multilevel logistic regression was used to assess the degree of neighborhood (i.e. block group) variation in sidewalk unevenness, sidewalk obstruction and the presence of physical disorder and the association with area-level characteristics.

Results: 1780 street segments were audited. Block groups that were predominantly African-American were 38 times more likely to have a lot of unevenness, 15 times more likely to have many obstructions, and 12 times more likely to have physical disorder. Poverty rate was not independently associated with sidewalk walkability; however, block groups with the highest poverty rates were 21 times more likely to have physical disorder.

Conclusion: The results indicate that aspects of the built environment vary by characteristics of the neighborhood. This suggests that there is a differential investment in community infrastructures and resources in neighborhoods that are mostly African-American. This differential investment is likely to influence disparities in rates of physical activity.
\end{abstract}

$\mathrm{R}$ egular physical activity reduces the risk of various diseases. ${ }^{1}$ Despite the well-known benefits of physical activity, 23\% of American adults report no physical activity during their leisure time. ${ }^{2}$ Among adults who report participating in some physical activity, 53\% do not engage in enough physical activity to reduce health risks. ${ }^{2}$ In addition, there are disparities in rates of physical activity with $61 \%$ of AfricanAmerican adults (compared with 50\% of white adults) and $63 \%$ of individuals in the lowest income group (compared with 50\% of individuals in the highest income group) not meeting physical activity recommendations. ${ }^{2}$

Walking is a basic human behavior and has been publicised recently as an excellent way to achieve the recommended amount of daily physical activity. ${ }^{3}$ It is an activity that most people can engage in, regardless of age or fitness level. Moreover, it is an activity that does not require special skills or equipment and, theoretically, can be performed within one's neighborhood.

There is a growing body of evidence to suggest that the environment in which people live can influence walking. For example, people walk more in communities that have sidewalks in good condition with few obstructions, provide destinations and facilities that can be reached within walking distance and are free from physical disorder (eg trash, abandoned buildings). ${ }^{4-7}$ Not all neighborhoods or environments provide the same opportunities to engage in walking for either transportation or recreational purposes. Individuals living in low-income and racial/ethnic minority communities experience disproportionate access to environmental features that support physical activity. ${ }^{8}$ This disproportionate access may be associated with the disparities in physical activity rates.

Most of the research assessing the difference in environments by race and income, however, has focused on perceived

access to places to be physically active as opposed to observed access. Although the use of community audits to observe characteristics of the built environment has increased, there is a lack of studies assessing differences in observed characteristics of street-scale environments (eg sidewalks) by area-level factors (eg poverty level and racial distribution). Street-scale data capture detailed information about an individual's street, an important consideration when developing interventions to encourage individuals to walk more.

The purpose of this study was to assess the extent to which area-level factors, poverty rate and racial distribution are associated with aspects of the street-scale environment using community audits. In particular, the objective of this study was to: (a) examine the extent of the spatial variation of walkability (unevenness and obstruction) of sidewalks and the presence of physical disorder; (b) determine the association of walkability and physical disorder with the contextual effect of block-group poverty rate and racial distribution; and (c) determine whether poverty rate and racial distribution explain any spatial differences in walkability and physical disorder.

\section{METHODS}

This study was an ancillary of the Garden of Eden (GOE) project, which was located in the St Louis metropolitan area. GOE was a quasi-experimental study aimed at increasing fruit and vegetable consumption through a community-run produce market among low-income, African-American adults. ${ }^{9}$ We used the location of GOE participants to select street segments

Abbreviations: DIC, deviance information criterion; $\mathrm{GOE}$, Garden of Eden; ICC, intraclass correlation; IOR, interval odds ratio; MOR, median odds ratio 
nested within block groups. A street segment was defined as a street between two intersections, including both sides. A random sample of street segments stratified by block group poverty rate $(<10 \%, 10-19 \%, 20 \%$ or higher $)$ and racial distribution (proportion of African-American residents $>50 \%$, proportion of white residents $>50 \%$ ) was selected as comparisons from both the city and the adjacent county. All selected street segments were subsequently audited for walkability and physical disorder.

\section{Block group selection}

Block group poverty rate and racial distribution were obtained from the 2000 United States census. Only predominantly African-American or white block groups were considered in the current study because $95 \%$ of the population residing in the study area self-identified as either African-American or white. As suggested by previous research, we used the percentage of the population living below the United States federal poverty line as a measure of area socioeconomic position and divided the block groups into high ( $\geqslant 20 \%)$ medium ( $10-19 \%)$ and low $(<10 \%)$ poverty. ${ }^{1011}$ The study area comprised 898 block groups. A stratified random sample of 210 block groups was selected, with 60 from block groups with at least 20\% of individuals living in poverty and a proportion of AfricanAmerican residents greater than 50\% (because GOE targeted African-American adults living in predominantly high poverty areas) and 30 from each remaining strata (table 1).

\section{Street segment selection}

Twenty-five per cent of the street segments nested within the block groups were randomly selected to be audited from each block group that had a minimum of five street segments. All street segments were audited in block groups with five or fewer segments. In an effort to capture residential street characteristics, only street segments that were considered $50 \%$ or more residential were audited. Residential was defined as more than half of the street segment composed of commercial properties (eg stores, restaurants). Such street segments were eliminated and a replacement segment was audited.

\section{Community audit tool development}

The audit tool used in this study was developed by selecting pertinent items from a tool measuring features of the streetscale environment related to recreational and transportationbased physical activity. ${ }^{12}$ The items were selected on the basis of their interobserver reliability (kappas ranging from 0.62 to 0.90 ) and ability to capture variation across street segments. ${ }^{12}$

Our audit tool assessed the presence and walkability of sidewalks and the presence of physical disorder. The presence of sidewalks was measured as no sidewalk on either side of the street, sidewalk on one side of the street or sidewalk on both sides of the street. In the analysis, the presence of sidewalks was dichotomised into one or both sides of the street versus no sidewalks present. The walkability of the sidewalks was measured using two items, one assessing the levelness and condition of sidewalks (eg alignment, cracks, broken sections, weeds) and one assessing the amount of artificial or natural obstructions on sidewalks (eg garbage cans, cars, trees). Each of these items was measured on a four-point scale, ranging from none (1) to a lot (4). In the analysis, levelness and condition of the sidewalks was dichotomised into "a lot or some unevenness" versus "a little or no unevenness". Sidewalk obstructions were dichotomised into "a lot or some obstructions" versus "a little or no obstructions".

Three items assessed the physical disorder present on a street segment. The first item was the number of abandoned buildings or vacant lots on the street segment. This item was a continuous variable that was categorised into none, a little (one abandoned building or vacant lot), some (two or three) or a lot (four or more) based on the distribution of the data (ranging from 0 to 28 , mean of 0.8 ). The next two items measured the presence of trash and graffiti, broken windows or abandoned cars on the street segment. Each of these items was measured on a four-point scale, ranging from none ( 1 ) to a lot (4). In the analysis, we dichotomised the presence of physical disorder into "any" versus "none".

Pairs of trained auditors walked the selected streets during daylight hours (from 08:00 to 17:00 hours) at varying times of the day and days of the week. A comprehensive protocol for data collection was used to train auditors, which included operational definitions of the response scale for each item. ${ }^{12}$ All audits were conducted between August 2004 and January 2005.

\section{Statistical analysis}

We used a multilevel logistic model to examine the spatial variation in sidewalk walkability and physical disorder with street segments nested within block groups. In the analyses, we controlled for age of housing stock (cut-off post-1945 housing; 2000 United States Census) and location (city versus county) of the block groups to account for the difference in age of sidewalks, which may influence the condition.

We used restricted iterative generalised least squares ${ }^{13}$ and Markov chain Monte Carlo methods. In the multilevel models, the random components were assessed at the individual level and the block group level. We found no evidence of extra binomial variation using chi-square tests in an empty model, suggesting that the logistic model is appropriate.

To determine the spatial variation in sidewalk walkability and physical disorder among block groups and its relative importance to block group poverty rate and racial distribution, we calculated three measures: (1) the intraclass correlation (ICC); (2) median odds ratio (MOR); and (3) interval odds ratio (IOR). The ICC is the percentage of the total variance between block groups, namely ICC $=\left[V_{\mathrm{n}}\right] /\left[V \mathrm{n}+V_{\mathrm{i}}\right] \times 100$, where $V_{\mathrm{n}}=$ block group variance and $V_{\mathrm{i}}=\pi^{2} / 3 .{ }^{13} \mathrm{~A}$ high ICC indicates large spatial variance between block groups.

The MOR is defined as the median value of the odds ratio between a block group most likely to have poor walkability and

Table 1 Number of block groups audited by racial distribution and poverty rate $(\mathrm{n}=210)$

\begin{tabular}{lll}
\hline & $\begin{array}{l}\text { Proportion of } \\
\text { African-American residents } \\
>\mathbf{5 0 \%}\end{array}$ & $\begin{array}{l}\text { Proportion of } \\
\text { white residents } \\
>\mathbf{5 0} \%\end{array}$ \\
\hline Percentage of residents living in poverty & 30 & 30 \\
Low poverty rate $(<10 \%)$ & 30 & 30 \\
Medium poverty rate $(10-19 \%)$ & $60^{*}$ & 30 \\
High poverty rate $(\geqslant 20 \%)$ & & \\
\hline
\end{tabular}

*The number of block groups considered African-American, high poverty is higher because a majority of Garden of Eden participants resided in these block groups and we wanted to ensure the capture of most of the block groups with Garden of Eden participants. 
the presence of physical disorder and the block group least likely to have poor walkability and the presence of physical disorder when randomly picking out two block groups. ${ }^{14}$ The MOR is the increase in likelihood a street segment would have if it were instead located in a block group with a higher likelihood of poor sidewalk walkability or with physical disorder. The MOR is calculated as:

$$
M O R \approx \exp \left(95.0 \sqrt{V_{n}}\right)
$$

where

$$
\sqrt{V_{n}}
$$

is the block group-level variance. If the MOR were 1.0, there would be no differences among block groups in the probability of having poor sidewalk walkability or physical disorder. If there were strong differences among block groups, the MOR would be large and the use of block groups would be important in order to understand the probability of having poor sidewalk walkability or physical disorder.

The IOR provides for a comparison of the importance of the block group poverty rate (racial distribution) compared with the variation remaining among block groups. The $\mathrm{IOR}_{80}$ is defined as the interval centered on the median of the distribution that comprises $80 \%$ of the values of the odds ratio. The $\mathrm{IOR}_{80}$ is calculated as follows:

$$
I O R \approx \exp \left(\beta \pm 1.81 \sqrt{V_{n}}\right)
$$

where $\beta$ is the parameter estimate of the block group poverty rate (racial distribution) and

$$
\sqrt{V_{n}}
$$

is the block group-level variance. If the residual variation among block groups is small, the interval will be narrow. If the interval contains the value of one, the effect of block group poverty rate (racial distribution) is not very strong compared with the remaining residual block group variation.

We also calculated traditional odds ratios associated with all block group-level variables. Based on Markov chain Monte Carlo models, we calculated 95\% credible intervals for all MOR, IOR, and odds ratios using the posterior distribution of the area variance. ${ }^{14}$ Multilevel models were developed and fit using the multilevel modeling software MLwiN, version 2.0.2. ${ }^{15}$ We calculated the deviance information criterion (DIC) for each model as a measure of model fit. A model with its DIC being at least 3 points lower than a second model is considered to have a better fit. ${ }^{16}$

\section{RESULTS}

\section{Sidewalk unevenness}

A total of 1780 street segments within 210 block groups were audited. After aggregating to the block group level, 635 street segments $(35.7 \%)$ did not have sidewalks on either side of the street or did not have valid data. These street segments were excluded from the analysis assessing sidewalk unevenness and obstructions leaving 1140 street segments available for analysis. Of street segments with sidewalks, $12.6 \%$ were reported as having a lot of unevenness (table 2). Poverty rate and racial distribution was not available for 18 street segments.

Of the total variance, $74.6 \%$ was between block groups based on an empty logistic model (table 3). The MOR was 14.24 (95\% credible interval $7.77 ; 34.18$ ) suggesting large spatial variation between block groups in sidewalk unevenness. After controlling for the age of housing stock and living in the city of St Louis or the adjacent county, the ICC was reduced to $67.4 \%$, indicating that both variables explained $13.2 \%$ of the variance in sidewalk unevenness between block groups. Next, we added poverty rate to the logistic model and the ICC and MOR were similar to the model without this variable. This suggests that poverty rate was unable to explain much of the variance in sidewalk unevenness between block groups. Next, we added whether a block group was predominantly African-American or white. This reduced the ICC and MOR to $61.1 \%$ and 8.68, respectively. Block groups that were predominantly African-American were 29.69 times more likely to have street segments with a lot of unevenness. The IOR for both block group racial distribution and poverty rate were large, suggesting that other factors should be considered in understanding differences in block group-level sidewalk unevenness.

\section{Sidewalk obstruction}

Of the 1780 street segments, 635 segments did not have sidewalks on either side of the street and no valid data were available for three segments, leaving 1138 street segments available for analysis. Of the total variance, $56.8 \%$ was between block groups based on an empty logistic model (table 4 ). The MOR (7.22) and ICC (56.8\%) suggested large spatial variation between block groups in sidewalk obstruction. Controlling for the age of housing stock and living in the city of St Louis or the adjacent county reduced the ICC by $7.5 \%$. Next, we added poverty rate to the logistic model, which increased the spatial variation between block groups based on the increased ICC and MOR. Adding racial distribution did not contribute to the model; however, block groups in which the population was predominantly African-American were 15.05 times more likely to have street segments that were observed to have a lot of obstruction after controlling for the age of housing stock and block group location. The model containing both racial distribution and poverty rate showed that only $11.5 \%$ of the variance between block groups in sidewalk obstruction was explained by these variables. The IOR for both variables were large

Table 2 Area characteristics for sidewalk condition and physical disorder

\begin{tabular}{lcll}
\hline & $\begin{array}{l}\text { Sidewalk unevenness } \\
\text { (a lot) }(\mathbf{n}=1122)\end{array}$ & $\begin{array}{l}\text { Sidewalk obstruction } \\
\text { (a lot) }(\mathbf{n}=1120)\end{array}$ & $\begin{array}{l}\text { Physical disorder } \\
\text { (any) }(\mathbf{n}=1780)\end{array}$ \\
\hline $\begin{array}{l}\text { Total } \\
\text { Poverty rate }\end{array}$ & $141(12.6 \%)$ & $65(5.8 \%)$ & $933(53.0 \%)$ \\
$<10 \%$ & $9(2.9 \%)$ & $10(3.2 \%)$ & $224(31.6 \%)$ \\
$10-19 \%$ & $34(11.5 \%)$ & $17(5.7 \%)$ & $238(53.04 \%)$ \\
$20+\%$ & $98(19.0 \%)$ & $38(7.4 \%)$ & $471(78.0 \%)$ \\
$\begin{array}{l}\text { Racial distribution } \\
>50 \% \text { African-American }\end{array}$ & $126(20.6 \%)$ & $54(8.8 \%)$ & $618(67.9 \%)$ \\
$>50 \%$ White & $15(3.0 \%)$ & $11(2.2 \%)$ & $315(37.0 \%)$ \\
\hline
\end{tabular}


Table 3. Measures of association and variation in sidewalk unevenness (a lot versus some/none) based on multilevel logistic regression*

\begin{tabular}{|c|c|c|c|c|c|}
\hline & Empty model & $\begin{array}{l}\text { Model with } \\
\text { age of housing } \\
\text { stock and block } \\
\text { group location }\end{array}$ & $\begin{array}{l}\text { Model with } \\
\text { poverty rate }\end{array}$ & $\begin{array}{l}\text { Model with } \\
\text { racial } \\
\text { distribution }\end{array}$ & $\begin{array}{l}\text { Model with } \\
\text { poverty rate } \\
\text { and racial } \\
\text { distribution }\end{array}$ \\
\hline \multicolumn{6}{|l|}{$\begin{array}{l}\text { Measures of association } \\
\text { Odds ratio }\end{array}$} \\
\hline Poverty rate $10-19 \%$ vs $<10^{\circ}$ & & & $4.30(0.63-25.00)$ & & $4.46(0.92-27.71)$ \\
\hline Poverty rate $20+\%$ vs $<10 \%$ & & & $7.55(1.03-47.17)$ & & $4.52(0.95-33.30)$ \\
\hline$>50 \%$ AA vs $>50 \%$ white & & & & $37.90(10.26-249.20)$ & $29.69(9.80-103.43)$ \\
\hline \multicolumn{6}{|l|}{ Interval odds ratio } \\
\hline Poverty rate $10-19 \%$ vs $<10$ & & & $0.03-551.76$ & & $0.07-273.37$ \\
\hline Poverty rate $20+\%$ vs $<10 \%$ & & & $0.06-969.25$ & & $0.07-277.22$ \\
\hline$>50 \%$ AA vs $>50 \%$ white & & & & $0.58-2464.81$ & $0.48-1819.70$ \\
\hline \multicolumn{6}{|l|}{$\begin{array}{l}\text { Measures of variation or } \\
\text { clustering }\end{array}$} \\
\hline Block group variance & 7.8199 & 6.7879 & 7.1967 & 5.3197 & 5.1723 \\
\hline PCV $\%$ & N/A & N/A & -6.0 & -21.6 & -23.8 \\
\hline Median odds ratio ( $95 \%$ Crl) & $14.24(7.77-34.18)$ & $11.88(6.62-22.58)$ & $12.79(6.90-28.31)$ & $8.95(4.93-18.28)$ & $8.68(5.16-16.79)$ \\
\hline ICC \% & 74.6 & 67.4 & 68.6 & 61.8 & 61.1 \\
\hline $\mathrm{DIC}$ & 579.76 & 571.28 & 546.29 & 535.05 & 535.01 \\
\hline
\end{tabular}

AA, African-American; $\mathrm{Crl}$, credible interval; DIC, deviance information criterion; ICC, intraclass correlation; PCV, proportional change in variance relative to model with age of housing stock and block group location.

*All models except the empty model contain the variables of age of housing stock and block group location in St Louis City or County.

suggesting that other variables need to be considered to understand differences in block group-level sidewalk obstruction.

\section{Physical disorder}

Of the 1780 street segments, data were available for 1762 segments. Of the total variance, $69.9 \%$ was between block groups (table 5). The MOR (13.80) and ICC (69.9\%) suggest large spatial variation between block groups in physical disorder. Controlling for the age of housing stock and living in the city of St Louis or the adjacent county reduced the ICC only marginally. Next, we added poverty rate to the logistic model, which explained $15.3 \%$ of the variance in disorder between block groups. The racial distribution at the block group level reduced the spatial variation in disorder to an ICC of $61.5 \%$, but important spatial variation remained based on the median and IOR. The model containing poverty rate and racial distribution explained $28.3 \%$ of the variance in disorder. Again, poverty rate and racial distribution explained only a portion of the variation in street segment disorder.

\section{DISCUSSION}

Previous research suggests that characteristics of the built environment, such as walkability and disorder, influence rates of walking and overall physical activity behavior. This study builds on previous research by demonstrating that some of the variability in characteristics of block groups is explained by racial distribution and poverty rates. This variability in block groups may contribute to disparities in rates of physical activity behavior. There remains, however, a large amount of variability unaccounted for between block groups. Some research has shown that the social environment (eg fear of crime, neighborhood social cohesion) influences physical activity. ${ }^{17-19}$ Therefore, interventions to address physical inactivity will need to consider more than the physical environment and the possibility of an

Table 4 Measures of association and variation in sidewalk obstruction (a lot versus some/none) based on multilevel logistic regression*

\begin{tabular}{|c|c|c|c|c|c|}
\hline & Empty model & $\begin{array}{l}\text { Model with } \\
\text { age of housing stock } \\
\text { and block group } \\
\text { location }\end{array}$ & $\begin{array}{l}\text { Model with } \\
\text { poverty rate }\end{array}$ & $\begin{array}{l}\text { Model with } \\
\text { racial distribution }\end{array}$ & $\begin{array}{l}\text { Model with } \\
\text { poverty rate and } \\
\text { racial distribution }\end{array}$ \\
\hline \multicolumn{6}{|l|}{ Measures of association } \\
\hline Poverty rate $10-19 \%$ vs $<10 \%$ & & & $1.38(0.33-6.00)$ & & $0.85(0.15-3.96)$ \\
\hline Poverty rate $20+\%$ vs $<10 \%$ & & & $1.46(0.25-8.07)$ & & $0.55(0.06-3.23)$ \\
\hline$>50 \%$ AA vs $>50 \%$ white & & & & $15.05(4.18-77.32)$ & $13.69(4.15-51.58)$ \\
\hline \multicolumn{6}{|l|}{ Interval odds ratio } \\
\hline Poverty rate $10-19 \%$ vs $<10 \%$ & & & $0.03-67.50$ & & $0.03-25.76$ \\
\hline Poverty rate $20+\%$ vs $<10 \%$ & & & 0.03 to 74.45 & & $0.02-16.55$ \\
\hline$>50 \%$ AA vs $>50 \%$ white & & & & $0.42-543.54$ & $0.45-413.86$ \\
\hline \multicolumn{6}{|l|}{ Measures of variation or clustering } \\
\hline Block group variance & 4.3278 & 4.0050 & 4.6197 & 3.9263 & 3.5465 \\
\hline PCV \% & N/A & N/A & 6.7 & -2.0 & -11.5 \\
\hline Median odds ratio (95\% Crl) & $7.22(3.76-13.83)$ & ) $\quad 6.69(3.49-13.30)$ & $7.71(3.99-16.36)$ & $6.57(3.43-15.35)$ & $5.98(3.38-10.16)$ \\
\hline ICC \% & 56.8 & 54.9 & 58.4 & 54.4 & 51.9 \\
\hline $\mathrm{DIC}$ & 416.78 & 416.40 & 403.44 & 392.85 & 394.65 \\
\hline
\end{tabular}


Table 5 Measures of association and variation in disorder (any versus none) based on multilevel logistic regression*

\begin{tabular}{|c|c|c|c|c|c|}
\hline & Empty model & $\begin{array}{l}\text { Model with } \\
\text { age of housing stock } \\
\text { and block group } \\
\text { location }\end{array}$ & $\begin{array}{l}\text { Model with } \\
\text { poverty rate }\end{array}$ & $\begin{array}{l}\text { Model with } \\
\text { racial distribution }\end{array}$ & $\begin{array}{l}\text { Model with } \\
\text { poverty rate and } \\
\text { racial distribution }\end{array}$ \\
\hline \multicolumn{6}{|l|}{$\begin{array}{l}\text { Measures of association } \\
\text { Odds ratio }\end{array}$} \\
\hline Poverty rate $10-19 \%$ vs $<10 \%$ & & & $6.24(2.10-21.56)$ & & $5.39(2.08-14.13)$ \\
\hline Poverty rate $20+\%$ vs $<10 \%$ & & & 21.14 (6.45-99.09) & & $13.93(3.78-50.00)$ \\
\hline$>50 \%$ AA vs $>50 \%$ white & & & & $12.18(5.92-29.78)$ & $10.72(4.37-25.05)$ \\
\hline \multicolumn{6}{|l|}{ Interval odds ratio } \\
\hline Poverty rate $10-19 \%$ vs $<10 \%$ & & & $0.08-465.01$ & & $0.10-284.62$ \\
\hline Poverty rate $20+\%$ vs < $10 \%$ & & & $0.28-1575.09$ & & $0.26-735.95$ \\
\hline$>50 \%$ AA vs $>50 \%$ white & & & & $0.19-775.22$ & $0.20-566.32$ \\
\hline \multicolumn{6}{|l|}{ Measures of variation or clustering } \\
\hline $\begin{array}{l}\text { Block group variance } \\
\text { PCY \% }\end{array}$ & 7.635 & 6.696 & 5.673 & 5.265 & 4.804 \\
\hline PCV \% & N/A & N/A & -15.3 & -21.4 & -28.3 \\
\hline $\begin{array}{l}\text { Median odds ratio }(95 \% \mathrm{Crl}) \\
\text { ICC \% }\end{array}$ & $13.80(9.09-21.68)$ & $\begin{array}{l}11.68(7.83-18.02) \\
67.1\end{array}$ & $9.61(6.45-14.94)$ & $8.84(6.11-13.43)$ & $8.02(5.43-12.06)$ \\
\hline $\begin{array}{l}\text { ICC \% } \\
\text { DIC }\end{array}$ & $\begin{array}{r}69.9 \\
1540.0\end{array}$ & $\begin{array}{r}67.1 \\
1537.3\end{array}$ & $\begin{array}{r}63.3 \\
1519.3\end{array}$ & $\begin{array}{r}61.5 \\
1512.3\end{array}$ & $\begin{array}{r}59.4 \\
1508.3\end{array}$ \\
\hline
\end{tabular}

AA, African-American; $\mathrm{Crl}$, credible interval; DIC, deviance information criterion; ICC, intraclass correlation; PCV, proportional change in variance relative to model with age of housing stock and block group location.

*All models except the empty model contain the variables of age of housing stock and block group location in St Louis City or County.

interaction between the physical environment and the social environment.

The results of this study suggest there is a differential investment in community infrastructures and resources in neighborhoods that are mostly African-American or primarily low income. The objectives of this study were threefold. First, the extent of spatial variation of walkability of sidewalks and the presence of physical disorder was examined. Spatial variation was present for each of the walkability variables (i.e. unevenness and obstructions) as well as physical disorder between block groups. Second, the presence of an association between walkability and physical disorder and the contextual effect of block-group poverty rate and racial distribution was assessed. Poverty rate was unable to explain much of the variance in sidewalk walkability between block groups; however, racial distribution contributed independently to the variation in sidewalk walkability. Poverty rate and racial distribution contributed independently to the variation in physical disorder. Finally, spatial differences in walkability and physical disorder by poverty rate and racial distribution were assessed. Sidewalk unevenness and the number of natural or artificial obstructions are greater in neighborhoods that are predominantly African-American, whereas physical disorder is greater in neighborhoods that are predominantly AfricanAmerican and neighborhoods experiencing higher poverty rates than in primarily white neighborhoods with a lower percentage of individuals living in poverty.

\section{Limitations of the study}

First, in this study, we defined an individual's community as a block group. Defining an individual's community is difficult because it probably varies from person to person and

\section{What this paper adds}

Previous research has identified several characteristics of the physical environment that influence physical activity behavior. The results of this study suggest that differences exist in these characteristics (eg the quality and condition of sidewalks, presence of physical disorder) by area-level racial distribution and poverty rate. neighborhood to neighborhood. Moreover, the distance a person is willing to walk, for recreational or transportation purposes, is likely to be different depending on various individual characteristics. The block group is, however, the lowest geographical unit for which the United States Census tabulates and presents income and racial data. Statistical power is also maximised when examining neighborhoods (or, in our case, block groups) by using many block groups with few street segments. ${ }^{20}$ The method of using block groups, therefore, allows us to capture variability within a small geographical area and across many different areas.

Second, although the tool used previously demonstrated moderate reliability, capturing differences in neighborhood environments using auditors has several limitations. The condition of the physical environment may depend on the time of day, day of the week or month of the year. The auditors in this project started auditing in late summer when people are outside more and finished in the winter months when people tend to be inside more. The order of block groups audited was arbitrary and mixed (i.e. African-American and white as well as low-income and high-income block groups were audited during the same time periods). In addition, the auditors walked the street segments Monday to Saturday from 08:00 hours until dark. Although sidewalk conditions probably do not vary greatly on a day-to-day or week-to-week basis, the number of obstructions or the amount of physical disorder may depend on the time of day or season of the year. Previous research has assessed the potential effect of interviewer characteristics and found that locals rated neighborhood characteristics different from non-locals. ${ }^{21}$ Therefore, because we did not use local community members to audit, our results may be biased away from the null. As all segments were audited by individuals who had similar backgrounds the differences between block groups are, however, not likely to be a function of interviewer bias.

Third, whereas research suggests that the characteristics of the built environment, such as walkability and disorder, influence rates of walking and overall physical activity behavior, it is unclear from this study if the variability in block group walkability and physical disorder influence walking for recreational and/or transportation purposes. It is also unclear how other characteristics of neighborhood environments influence walkability and physical disorder. For example, an assessment of social disorder (eg the presence of gangs) may 


\section{Policy implications}

To address physical inactivity, policies and programmes need to be developed that ensure equal access to opportunities to engage in physical activity.

indicate that walkability and physical disorder is directly related to the presence of social disorder, and as such policies to improve neighborhood environments may need to start by addressing social disorder.

\section{Practical implications}

There are currently many efforts to address disparities in physical inactivity between African-American and white communities. Many efforts have, however, focused on changing individual behavior. The findings from this study suggest that efforts to address physical inactivity in African-American communities must address the inequality in environmental access to opportunities to be physically active. This is consistent with previous research that identified a lack of physical activity settings (eg parks) in low-income and racial/ethnic minority communities, a pattern referred to as deprivation amplification.

As is similar in other urban settings, the pattern of deprivation amplification in the St Louis area has been building over the past 50 years. With the advent of urban sprawl, many white, middleincome residents and businesses moved to suburban areas (i.e. St Louis County). This flight to the suburbs was the start of a shrinking tax base and subsequent financial difficulties in the city. The subsequent change in demographics (eg fewer people living in the city) contribute to the differential investment in community infrastructures. As such, efforts to increase physical inactivity need to address the geographical inequities that are reflective of broader social inequities based on race, income, culture and political power. Therefore, strategies should not only include improving sidewalks and cleaning up streets, but also developing policies to improve the economic stability in neighborhoods with few resources.

\section{ACKNOWLEDGEMENTS}

The authors would like to thank the Alvin J. Siteman Cancer Center at Barnes Jewish Hospital and Washington University School of Medicine in St Louis, Missouri, for the use of the Health Behavior Research Core, especially Jim Struthers for data management and selected statistical services. They also wish to thank Kylie Harrold and Chris Smith for their assistance with data collection.

\section{Authors' affiliations}

Cheryl M Kelly, Transtria, 3525 Watson Road, St Louis, MO 63139, USA

Elizabeth A Baker, Ellen K Barnidge, Amanda Lemes, Saint Lovis

University, School of Public Health, St Louis, MO 63104, USA
Mario Schootman, Department of Medicine and Pediatrics, Washington University School of Medicine, St Louis, MO, USA

Funding: This research was supported in part by grants from the American Cancer Society (TURPG-00-129-01-PBP), the Centers for Disease Control and Prevention (R06/CCR721356) and the National Cancer Institute (P30 CA91842).

Competing interests: None declared.

\section{REFERENCES}

1 Centers for Disease Control and Prevention. Physical activity and good nutrition: essential elements to prevent chronic diseases and obesity. Atlanta: Centers for Disease Control and Prevention, 2003.

2 Centers for Disease Control and Prevention. Behavioral risk factor surveillance system, 15 March 2006.http://www.cdc.gov/brfss (accessed Aug 2007).

3 Norman GJ, Mills PJ. Keeping it simple: encouraging walking as a means to active living. Ann Behav Med 2004;28:149-51.

4 Saelens BE, Sallis JF, Frank LD. Environmental correlates of walking and cycling: findings from the transportation, urban design, and planning literatures. Ann Behav Med 2003;25:80-91.

5 McCormack G, Giles-Corti B, Lange A, et al. An update of recent evidence of the relationship between objective and self-report measures of the physical environment and physical activity behaviours. J Sci Med Sport 2004;7:81-92.

6 Owen N, Humpel N, Leslie $\mathrm{E}$, et al. Understanding environmental influences on walking: review and research agenda. Am J Prev Med 2004;27:67-76.

7 Li F, Fisher KJ, Brownson R, et al. Multilevel modeling of built environment characteristics related to neighborhood walking activity in older adults. J Epidemiol Commun Health 2005;59:558-64.

8 Taylor WC, Carlos Poston W, Jones L, et al. Environmental justice: obesity, physical activity and healthy eating. J Phys Activity Health 2006;3(Suppl 1):S30-S54

9 Baker EA, Kelly C, Barnidge E, et al. The Garden of Eden: acknowledging the impact of race and class in efforts to decrease obesity rates. Am J Public Health 2006;96:1170-4.

10 Krieger N. Is breast cancer a disease of affluence, poverty, or both? The case of African-American women. Am J Public Health 2002;92:611-13.

11 Singh G. Area deprivation and widening inequalities in US mortality, 19691998. Am J Public Health 2003;93:1137-43.

12 Brownson R, Hoehner C, Brennan L, et al. Reliability of two instruments for auditing the environment for physical activity. J Phys Activity Health 2004; 1:189-207.

13 Goldstein H. Restricted unbiased iterative generalized least-squares estimation Biometrika 1989:76:622-3.

14 Merlo J, Chaix B, Ohlsson H, et al. A brief conceptual tutorial of multilevel analysis in social epidemiology: using measures of clustering in multilevel logistic regression to investigate contextual phenomena. J Epidemiol Commun Health 2006;60:290-7.

15 Rasbash J, Browne W, Healy M, et al. MLwiN Beta version 2.0. London: Institute of Education, University of London, 2003.

16 Spiegelhalter D, Best N, Carlin B, et al. Bayesian measures of model complexity and fit (with discussion). J Roy Statist Soc, Series B, Method 2002;64:583-616.

17 Altman DG, Feighery E, Robinson TN, et al. Psychosocial factors associated with youth involvement in community activities promoting heart health. Health Educ Behav 1998;25:489-500.

18 Brennan LK, Baker EA, Haire-Joshu D, et al. Linking perceptions of the community to behavior: are protective social factors associated with physical activity? Health Educ Behav 2003;30:740-55.

19 Wilson DK, Kirtland KA, Ainsworth BE, et al. Socioeconomic status and perceptions of access and safety for physical activity. Ann Behav Med 2004;28:20-8.

20 Snijders T, Bosker R. Multilevel analysis. An introduction to basic and advanced multilevel modeling. London: Sage Publications, 1999.

21 Andresen EM, Malmstrom TK, Miller DK, et al. Reliability and validity of observer ratings of neighborhoods. J Aging Health 2006;18:28-36. 